\title{
Is There a General Motor Program for Right Versus Left Hand Throwing in Children?
}

\author{
Jerry R. Thomas ${ }^{1 *}$, Jacqueline A. Alderson ${ }^{2}$, Katherine T. Thomas ${ }^{3}$, Amity C. Campbell ${ }^{2}$, W. Brent Edwards ${ }^{4}$, Stacey Meardon $^{4}$ and Bruce C. \\ Elliott ${ }^{2}$
}

${ }^{1}$ College of Education, University of North Texas, USA

${ }^{2}$ School of Sport Science, Exercise and Health, University of Western Australia, Australia

${ }^{3}$ Department of Kinesiology, Health Promotion, and Recreation, University of North Texas, USA

${ }^{4}$ Department of Kinesiology, lowa State University, USA

\begin{abstract}
The purpose of this study was to determine if a general motor program controlled some or all aspects of overhand throwing. Using a 12 camera Vicon motion analysis system to record data from body markers, a group of 30 Australian Aboriginal children 6-10 years of age threw with maximal effort into a large target area. Data were reduced and analyzed for numerous variables and correlations were calculated between dominant and non-dominant side variables that were deemed reliable. Results indicated that five variables showed significant dominant to non-dominant correlations. However, only two of the five were entered into both multiple regressions to predict horizontal ball velocity for the dominant vs. non-dominant sides. The variables entered suggested that more gross aspects of the movement (stride distance and pelvis flexion) were both correlated from dominant to non-dominant sides and predicted horizontal ball velocity. Thus, the general motor program does not appear to control the more complex and coordinated parts of the throwing motion.
\end{abstract}

Keywords: Central pattern generator; Dominant vs. non-dominate throwing; Motor programs

\section{Introduction}

The Central Nervous System (CNS) has been postulated to control task performance of a class of movements using pre-programmed strategies. These strategies comprise the general motor program (GMP), which can be adapted depending on choice of parameters [1]. Parameter choices typically include timing and force. However, it is possible that within the GMP, kinematics or joint position are not programmed. In a study of mirror writing, Latash [2] concludes that bilateral transfer, an indicator of the existence of a GMP, is accomplished by using external space variables, or task-specific motor learning. However, he also reported intrinsic variables associated with the tasks may provide input into an internal model used to coordinate movement [2].

Using pre-programmed strategies such as centrally planned hand trajectories and time scaling, the existence of the theoretical GMP has been supported in studies of simple two dimensional tasks involving hand movements. Similar hand path trajectories and velocity curves have been consistently shown in studies on hand movements between two targets [3-6]. However, the central control of this relatively simple task may not apply to complex three dimensional (3D) motions. In studies of 3D seated reaching movements involving large body motion, hand trajectories, joint kinematics and inter-joint coordination failed to meet the framework of the GMP $[7,8]$. This implies that centrally planned strategies may not exist for more complex movement patterns.

Bilateral transfer, another indicator of the existence of a GMP for a particular task, is the gain or loss in performance that occurs with the utilization of the unskilled limb [9]. Handwriting is the classic example of bilateral transfer. Multiple studies have demonstrated the motor equivalence, or similarities in style and spatial temporal pattern, when blindfolded participants write with dominant and non-dominant hands, as well as with the mouth [10-13]. Further evidence for bilateral transfer has been seen in wrist-twist tasks [14]. More recently, bilateral transfer has also been demonstrated in more complex task involving throwing. In a study of throwing, children who practiced throwing tasks demonstrated greater transfer of skill, when compared with a control group, in both immediate and delayed transfer tests [15]. Schmidt and Lee [1] suggested that specification of muscles or joints to be utilized in task performance is another parameter that can be specified by the motor program.

Throwing is a complex task requiring the intricate interaction of the body and the upper extremity and is performed at varying degrees of skill level. In a study of dominant and non-dominant overhand throwing in skilled adults, kinematic results failed to support the existence of a GMP [16]. On the dominant or skilled arm, kinematics varied by condition discounting the utilization of a central pattern. However, on the non-dominant or unskilled arm, joint patterns did vary with condition for some but not all variables examined, suggesting a reliance on a central pattern. The authors concluded that time scaling of joint position, a characteristic of a central pattern of control, does not occur in skilled throwing.

Interestingly in the above cited study, basic elements found in the dominant arm throws were present in the non-dominant throws [16]. The authors suggested when first learning to throw, participants use a central pattern to control joint kinematics; then, as skill is attained, this central control is overridden by a more complex pattern of movement exploiting degrees of freedom to optimize throwing outcomes. However, their study was limited to angular positions of arm segments

*Corresponding author: Jerry R. Thomas, College of Education, University of North TexasDenton, TX76203-5017, Tel: 940-565-2233; E-mail: Jerry.Thomas@unt.edu

Received August 10, 2011; Accepted August 27, 2011; Published August 29 2011

Citation: Thomas JR, Alderson JA, Thomas KT, Campbell AC, Edwards WB, et al. (2011) Is There a General Motor Program for Right Versus Left Hand Throwing in Children? J Biosens Bioelectron S1:001. doi: 10.4172/2155-6210.S1-001

Copyright: @ 2011 Thomas JR, et al. This is an open-access article distributed under the terms of the Creative Commons Attribution License, which permits unrestricted use, distribution, and reproduction in any medium, provided the original author and source are credited. 
and did not include trunk or lower extremity movements. Another possibility is the existence of a generalized motor program directing general features of throwing, such as trunk motion and step length, with the more complex patterns of upper extremity kinematics resulting from an interaction of the task, the thrower, and the environment.

The purpose of this study was to determine the relationship between dominant and non-dominant joint kinematics during over arm throwing in children. It was hypothesized that common features of dominant and non-dominant arm throwing suggest the existence of central representation, a key component of a motor program. The hypothesis was that the general features of overhand throwing, such as ball velocity and step length, would be related between dominant and non-dominant throws, but complex joint motions needed to fine tune throwing would not. This hypothesis was tested by examining joint kinematics during dominant and non-dominant maximal throws by children aged 6 to 10 years old.

\section{Materials and Method}

\section{Participants}

Mooditj Community College pupils [2] (an Australian Aboriginal school on the northern edge of the Perth metropolitan area) were recruited for this study. The sample $(N=30)$ consisted of 15 boys and 15 girls (see Table 1 for descriptive data). The investigation was approved by the Ethics and Human Research Committee of the University of Western Australia. The school principal and parents/guardians gave consent for participation of the children who gave their verbal consent. Participants were transported from their school to the Biomechanics Laboratory at the University of Western Australia for data collection.

\section{Procedures}

The procedures outlined herein (except for statistical analyses) were previously published in a paper examining age and gender differences in dominant arm throwing for Australian Aboriginal children [17].

Upon arrival participants had standard anthropometric measures of height and mass recorded. Children were asked if they played on organized teams in sports using overhand throwing (cricket, softball or tee ball). None reported playing on organized teams. Following three warm-up trials each participant completed a minimum of three maximal effort overhand throws at a large indoor goal (2.14 $\mathrm{m}$ high $\times$ $3.66 \mathrm{~m}$ wide) located $5 \mathrm{~m}$ in front of the release position. This procedure was completed for both dominant and non-dominate throwing arms. Participants were instructed to throw the tennis ball as hard as possible (e.g., with maximum velocity) with no further instructions or coaching provided. Visual observation suggested that participants did throw with maximal effort. Motion capture data of the participant and ball were recorded at $250 \mathrm{~Hz}$ with a 12 camera Vicon motion analysis system (Oxford Metrics Inc., UK). A customized University of Western Australia biomechanical model and marker set were used (based on the design of [18]. The marker set consisted of 38 retro reflective markers, $10 \mathrm{~mm}$ in diameter, fixed to the children's skin by adhesive double sided

\begin{tabular}{|l|l|l|l|l|l|l|}
\hline & \multicolumn{2}{l}{ Age (years) } & \multicolumn{2}{l|}{ Height $(\mathrm{cm})$} & \multicolumn{2}{l|}{ Mass $(\mathrm{kg})$} \\
\hline Gender & Mean & S.D. & Mean & S.D. & Mean & S.D. \\
\hline Girls $(\mathrm{n}=13)$ & 8.3 & 1.4 & 128.8 & 9.0 & 28.9 & 7.1 \\
\hline Boys $(\mathrm{n}=15)$ & 7.9 & 1.5 & 124.3 & 11.3 & 25.3 & 6.9 \\
\hline Combined $(\mathrm{N}=28)$ & 8.1 & 1.4 & 127 & 10 & 27.3 & 6.9 \\
\hline
\end{tabular}

Table 1: Demographic participant information.

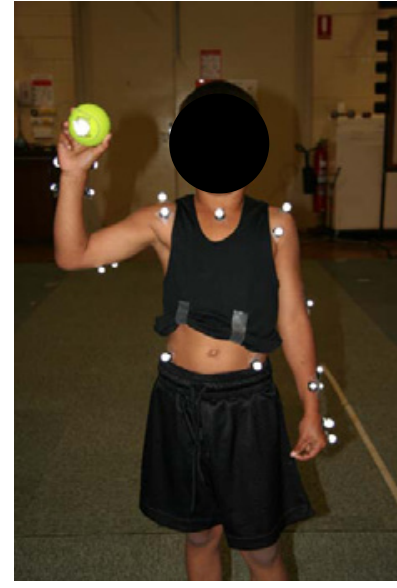

Figure 1: Reflective marker set used for calculation of joint kinematics.

non-allergenic tape (Figure 1). The tennis ball ${ }^{3}$ was defined with two retro reflective markers affixed to opposing sides of the ball. Prior to data analysis, a static trial was performed to establish local anatomical coordinate systems at each joint [4].

\section{Data reduction and modeling}

Each trial of interest was visually inspected to eliminate random marker movement incongruent with the motion being performed. The raw motion capture data were smoothed at $18 \mathrm{~Hz}$, determined via residual analysis, using a recursive digital Butterworth filter. The model used, which follows the standards outlined by the International Society of Biomechanics [19], was written using Body Builder software (Oxford Metrics, Inc.) and allowed for the determination of 3D kinematic data. The model outputs 3D motion for all degrees of freedom at the wrist, elbow, shoulder, trunk and pelvis segments. Segment motion can be described relative to the global laboratory coordinate system correlative to another segment (joint angles). Joint angles are referenced to the anatomical position, with the determination of each axis displacement calculated in an ordered series of rotations, namely: flexion/extension, adduction/abduction, internal/external rotation. Uniquely, the shoulder angles were determined by a polar angle decomposition, the current standard method to represent shoulder joint kinematics in throwing [20].

Stride length and horizontal ball velocity were calculated; kinematic variables determined at ball release included trunk flexion (upper torso segment relative to the pelvis segment), elbow flexion (forearm segment relative to the upper arm segment),as well as shoulder horizontal adduction and shoulder abduction (upper arm segment relative to the thorax segment); peak kinematic variables determined during the throwing motion included axial rotation (separation angle between the upper torso segment relative to the pelvis segment) and peak shoulder external rotation (upper arm segment relative to the thorax segment). In addition, the corresponding peak angular velocities where calculated for each of the kinematic variables listed above, except stride length. We refer to separation angular velocity as closing velocity (an axial rotation measure). The 3D ball trajectory was used to determine the forward horizontal velocity component via standard differentiation procedures. Data were analyzed from the start of the throwing motion (initial pelvis forward rotation) through ball release to one second [5] following release. The data were temporally normalized to 100 points to allow for between participant comparisons. Kinematic averages for 
discrete time points at ball release and peak values over the throwing motion were calculated.

\section{Statistical analysis}

Data were visually analyzed for errant data points prior to statistical analyses. All statistical analyses were performed in SPSS following screening for normality and outliers. Reliability of kinematic variables and horizontal ball velocity between trials were evaluated using Intraclass correlation (ICC) estimates. Only those variables meeting an inclusion criterion of an ICC greater than 0.70 were used for further statistical analysis. Simple linear regression was used to compare dominant throwing variables to non-dominant throwing variables. The criterion alpha level was set to 0.05 . In addition, stepwise multiple regressions were used to predict horizontal ball velocity from kinematic variables for both dominant and non-dominant throwing. The $F$ probability for entry and removal was 0.05 and 0.10 respectively.

\section{Results}

Of the kinematic variables meeting the inclusion criterion of an intraclass reliability estimate greater than $70^{6}$ only five displayed significant correlations overall between right and left side kinematic variables. These variables included: ball velocity, stride length, trunk flexion at ball release, shoulder horizontal adduction at ball release, and peak elbow flexion velocity during the throwing motion (Table 2). The other measures reported in Table 2-elbow flexion, shoulder external rotation, two axial rotation measures (separation angle and closing velocity), and shoulder abduction velocity-were not correlated between the right and left sides.

Multiple regressions for the dominant arm throwing identified three predictor variables for horizontal ball velocity. These predictor variables including stride length, trunk flexion, and shoulder horizontal adduction accounted for $80 \%$ of the variance in ball velocity (See Figure 2; $F(3,23)=31.66, p<0.001)$. Stride length and trunk flexion were also identified as significant predictor variables for non-dominant arm throwing horizontal ball velocity. Together, these two variables accounted for $49 \%$ of the variance in ball velocity (See Figure 2; F (2, 25) $=12.04, p<0.001$ ).

\begin{tabular}{|c|c|c|c|c|}
\hline \multirow[b]{2}{*}{ Variable } & \multirow{2}{*}{$\begin{array}{l}\text { Right } \\
\text { Mean (SD) }\end{array}$} & \multirow{2}{*}{$\begin{array}{l}\text { Left } \\
\text { Mean (SD) }\end{array}$} & \multirow{2}{*}{\multicolumn{2}{|c|}{$r$}} \\
\hline & & & & \\
\hline Trunk flexion $\left({ }^{\circ}\right)$ & $-1.4(10.4)$ & $-5.5(12.8)$ & 0.68 & ** \\
\hline Ball Velocity (m/s) & $14.0(2.8)$ & $10.8(1.5)$ & 0.62 & ** \\
\hline Stride $(\mathrm{cm})$ & $481.7(198.4)$ & $394.9(355.0)$ & 0.46 & * \\
\hline Shoulder horizontal adduction $\left({ }^{\circ}\right)$ & $27.3(12.1)$ & $39.4(13.0)$ & 0.40 & * \\
\hline Elbow flexion velocity ( $\% / s)$ & $-1045.3(217.0)$ & $-909.2(157.2)$ & 0.40 & * \\
\hline Elbow flexion $\left({ }^{\circ}\right)$ & $49.5(20.0)$ & $48.7(15.5)$ & 0.35 & \\
\hline Shoulder external rotation $\left({ }^{\circ}\right)$ & $135.3(18.9)$ & $125.4(13.5)$ & 0.34 & \\
\hline Shoulder abduction $\left({ }^{\circ}\right)$ & $89.6(12.2)$ & $109.5(9.9)$ & 0.26 & \\
\hline Separation angle $\left(^{\circ}\right)$ & $30.8(11.4)$ & $29.1(10.0)$ & 0.23 & \\
\hline Closing velocity $(\% / s)$ & $-281.6(88.4)$ & $-287.3(85.2)$ & 0.17 & \\
\hline Shoulder abduction velocity $\left({ }^{\circ} / \mathrm{s}\right)$ & $-103.6(66.2)$ & $-82.7(71.3)$ & -0.08 & \\
\hline
\end{tabular}

Table 2: Overall means, standard deviations (SD), and dominant to non-dominant arm correlations for reliable (Intraclass Correlation $>0.70$ ) dominant and nondominant overhand throwing variables. Asterisks indicate significant correlation, $r\left(\mathrm{p}<0.05^{*}, \mathrm{p}<0.01^{* *}\right)$.

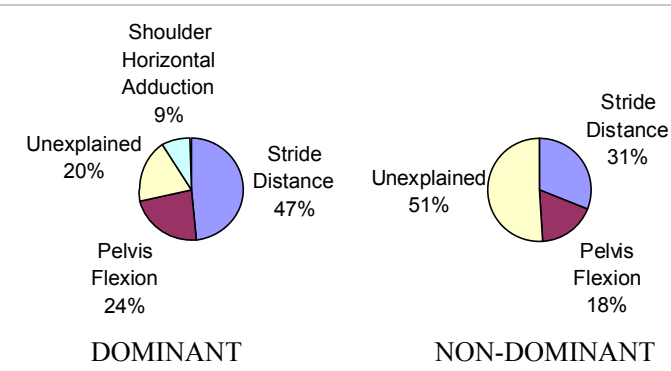

Figure 2: Predictors of ball velocity $\left(\mathrm{r}^{2}\right)$ for multiple regression analysis.

\section{Discussion}

The purpose of this study was to determine the relationship between dominant and non-dominant kinematic parameters during overhand throwing in children. It was hypothesized that general movement patterns such as stride length and trunk flexion would be significantly correlated, while more complex joint patterns of the upper extremity would be less correlated.

A moderate correlation was observed between dominant and nondominant horizontal ball velocity. Either anthropometrics account for this relationship and the larger stronger subjects throw faster in general, or there exists bilateral transfer for the throwing task. When the variance in ball velocity, due to height and weight were controlled using hierarchical procedures, the correlation between dominant and non-dominant horizontal ball velocity remained significant $(r=$ $0.50, d f=25, p<0.001)$. This suggests that bilateral transfer is indeed a determining factor for non-dominant horizontal ball velocity.

The highest correlations between dominant and non-dominant throwing were observed for stride step length and trunk flexion. Multiple regressions suggested that step length and trunk flexion were also key predictors of ball velocity in both dominant and non-dominant throws. It is likely that these general features are task parameters of an underlying motor program.

Elbow flexion velocity and shoulder horizontal adduction at release were also significantly correlated between dominant and non-dominant throws. Unlike the general features of step length and trunk flexion, these parameters did not predict ball velocities in either the dominant or the non-dominant throws. Thus, it is unlikely that these two variables are task parameters associated with the GMP. Elbow flexion velocity could be significantly correlated due to body anthropometrics. However, when height and weight were controlled by hierarchical procedures, the relationship between dominant and non-dominant remained significant $(r=0.39, d f=25, p<0.05)$. It is possible that the elbow is not as complex a joint as the shoulder and thus may be more easily manipulated to enhance performance in unskilled throwing.

Previous research suggests that horizontal adduction is not an invariant characteristic in overhand throwing $[3,16]$. Consistent with this, the current study reveals a moderate correlation for horizontal adduction at ball release between dominant and non-dominant arms suggesting an association with a GMP. However, horizontal adduction at release only predicts ball velocity in the dominant or skilled arm. Stodden, Langendorfer, Fleisig, and Andrews [21] in a study of kinematics associated with skill acquisition of throwing, reported that shoulder horizontal adduction is a key kinematic variable associated with skill development. In agreement with their findings, more skilled children demonstrated less horizontal adduction at ball release. 
Citation: Thomas JR, Alderson JA, Thomas KT, Campbell AC, Edwards WB, et al. (2011) Is There a General Motor Program for Right Versus Left Hand Throwing in Children? J Biosens Bioelectron S1:001. doi: 10.4172/2155-6210.S1-001

Regression analysis reveals more unexplained error exists in the non-dominant arm. While stride distance and trunk flexion are key predictors of ball velocity in unskilled throwing, greater variability may minimize the expression of other kinematic predictors of ball velocity. Less variability in skilled throwing increases the likelihood of the discovery of key characteristics associated with ball velocity.

One limitation to this study is that some of the measured throwing characteristics that have previously been shown to influence throwing (e.g., wrist flexion/extension) were quite variable and thus, not reliable in these children's performance. Using them in the analysis under these conditions was not logical.

\section{Conclusion}

Overhand throwing is a complex task that requires the precise interaction of multiple joints and degrees of freedom in order to achieve optimal throwing velocity and accuracy. The results presented in this study suggest that bilateral transfer of an overhand throwing task may exist for general movement parameters, such as stride length and trunk flexion. These parameters are most likely features of an underlying GMP. Although moderate correlations were observed between dominant and non-dominant throwing for elbow flexion velocity and shoulder horizontal adduction, they were not found to be predictors of both dominant and non-dominant horizontal ball velocity. As such, the more complex movements of overhand throwing may be better explained from a dynamical systems perspective.

\section{References}

1. Schmidt RA, Lee LD (1999) Motor control and learning: A behavioral emphasis ( $3^{\text {rd }}$ ed), Human Kinetics, Champaign, IL.

2. Latash ML (1999) Mirror writing: Learning, transfer, and implications for internal inverse models. J Mot Behav 31: 107-111.

3. Abend W, Bizzi E, Morasso P (1982) Human arm trajectory formation. Brain 105: 331-348.

4. Atkeson CG, Hollerbach JM (1985) Kinematic features of unrestrained vertical arm movements. The J Neurosci 5: 2318-2330.

5. Hollerbach JM, Flash T (1982) Dynamic interactions between limb segments during planar arm movement. Biol Cybern 44: 67-77.

6. Soechting JF, Lacquaniti $F$ (1981) Invariant characteristics of a pointing movement in man. J Neurosci 1: 710-720.

7. Pozzo T, Stapley PJ, Papaxanthis C (2002) Coordination between equilibrium and hand trajectories during whole body pointing movements. Exp Brain Res 144: 343-350.

8. Thomas JS, Corcos DM, Hasan Z (2003) Effect of movement speed on limb segment motions for reaching from a standing position. Exp Brain Res 148: 377-387

9. Schmidt RA, Young DE (1987) Transfer of movmement control in motor learning. In Cormier SM, Hagman JD (Eds.) Transfer of learning, Academic Press, Orlando, FL.

10. Bruce D (1994) Lashley and the problem of serial order. American Psychol 49 93-104.

11. Keele SW, Cohen A, Ivry R (1990) Motor programs: Concepts and issues, In Jeannerod, M (Ed.) Attention and performance XIII. 77-110.

12. Merton PA (1972) How we control the contraction of our muscles, Scientific American 226: 30-37.

13. Rosenbaum DA, Cohen RG, Jax SA, Weiss DJ, van der Wel R (2007) The problem of serial order in behavior: Lashley's legacy,Hum Mov Sci 26: 525-554.

14. Shapiro DC (1977) Psychology of motor behavior and sport, Human Kinetics, Champaign, IL.
15. Liu J, Wrisberg CA (2005) Immediate and delayed bilateral transfer of throwing accuracy in male and female children,Research Quarterly for Exercise and Sport 76: 20-27.

16. Hore JJ, O'Brien MM, Watts SS (2005) Control of joint rotations in overarm throws of different speeds made by dominant and nondominant arms, $J$ Neurophysiol 94: 3975-3986.

17. Thomas JR, Alderson JA, Thomas KT, Campbell AC, Elliott BC (2010) Developmental gender differences for overhand throwing in Australian Aboriginal children, Research Quarterly for Exercise and Sport 81: 432-441.

18. Lloyd DG, Alderson J, Elliott BC (2000) An upper limb kinematic model for the examination of cricket bowling: A case study of Mutiah Muralitharan. J Sports Sci 18: 975-982.

19. Wu G, Cavanagh PR (1995) ISB recommendations for standardization in the reporting of kinematic data. J Biomech 28: 1257-1261.

20. Doorenbosch CA, Harlaar J, Veeger D (2003) The globe system: An unambiguous description of shoulder positions in daily life movements. $J$ Rehabil Res and Dev 40: 147-155.

21. Stodden DF, Langendorfer S J, Fleisig GS, Andrews JR (2006) Kinematic constraints associated with the acquisition of overarm throwing part II: Upper extremity actions. Research Quarterly for Exercise and Sport 77: 428-436.

22. Besier TF, Sturnieks DL, Alderson JA, Lloyd DG (2003) Repeatability of gait data using a functional hip joint centre and a mean helical knee axis. $\mathrm{J}$ biomech 36: $1159-1168$.

\section{Footnotes}

1. This research was supported by a Fellowship and Visiting Professor status to the first author from the University of Western Australia, as well as a Faculty Development Leave to the first and third authors from lowa State University.

2. Thanks to the children, parents, teachers, and educational leaders at Mooditj Community College for their participation and support of this study.

3. Tennis balls were selected for throwing because of the children's smaller hands and they provided no advantage/disadvantage if children had thrown other types of balls. While the markers on either side of the ball could be considered a limitation, observation of the children throwing suggested it did not interfere with the grip or throw.

4. The wrist joint center was defined as the midpoint between the radial and styloid process. The shoulder joint center was the intersecting point between a line from the anterior to posterior shoulder markers and a perpendicular line dropped from a marker placed on the acromion process. The elbow epicondyles were identified using the pointer method of joint cente identification [22]. The elbow joint centers were referenced to local reference frames during dynamic trials in order to create the dynamic anatomical joint centers.

5. The video time cycle was extended for 1 second beyond ball release to evaluate follow-through motion which did not influence performance, so was not included.

6. A number of variables that might be of interest could not be included because of low reliability estimates $(<.70)$ on the children (e.g., shoulder abduction, wrist flexion).

Submit your next manuscript and get advantages of OMICS Group submissions

Unique features:

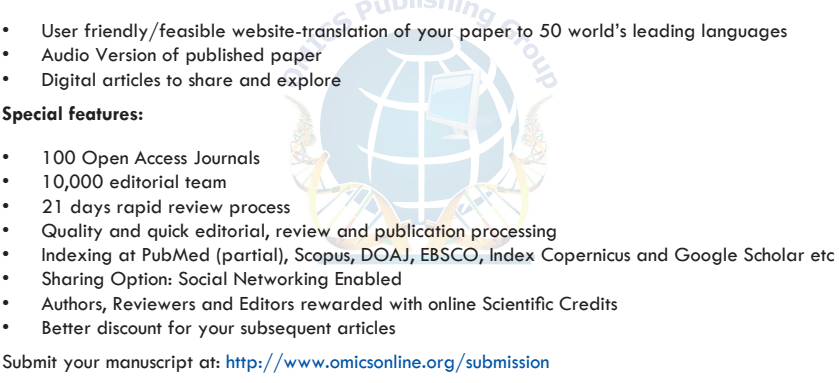

